UCRL-TR-209738

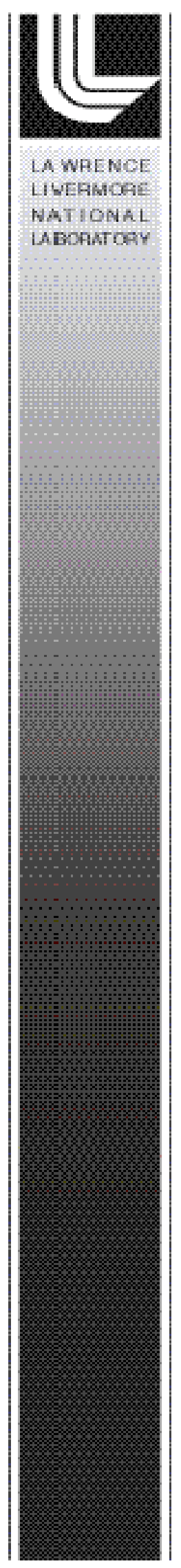

\title{
Modeling the Production of Beta-Delayed Gamma Rays for the Detection of Special Nuclear Materials
}

J. M. Hall (PI), J. A. Pruet, D. A. Brown, M.-A. Descalle and G. W. Hedstrom

Lawrence Livermore National Laboratory

S. G. Prussin

University of California at Berkeley

14 February 2005

Final Report submitted for NAI LDRD 04-ERD-042 


\section{Disclaimer}

This document was prepared as an account of work sponsored by an agency of the United States Government. Neither the United States Government nor the University of California nor any of their employees, makes any warranty, express or implied, or assumes any legal liability or responsibility for the accuracy, completeness, or usefulness of any information, apparatus, product, or process disclosed, or represents that its use would not infringe privately owned rights. Reference herein to any specific commercial product, process, or service by trade name, trademark, manufacturer, or otherwise, does not necessarily constitute or imply its endorsement, recommendation, or favoring by the United States Government or the University of California. The views and opinions of authors expressed herein do not necessarily state or reflect those of the United States Government or the University of California, and shall not be used for advertising or product endorsement purposes.

\section{Auspices Statement}

This work was performed under the auspices of the U. S. Department of Energy (DOE) by the University of California, Lawrence Livermore National Laboratory (LLNL) under Contract No. W-7405-Eng-48. The project (04-ERD-042) was funded by the Laboratory Directed Research and Development Program at LLNL. 


\title{
Modeling the Production of Beta-Delayed Gamma Rays for the Detection of Special Nuclear Materials
}

\author{
J. M. Hall (PI), J. A. Pruet, D. A. Brown, M.-A. Descalle and G. W. Hedstrom
}

Lawrence Livermore National Laboratory, Livermore, CA 94550

\author{
S. G. Prussin
}

University of California, Berkeley, CA 94720

\begin{abstract}
The objective of this LDRD project was to develop one or more models for the production of $\beta$-delayed $\gamma$ rays following neutron-induced fission of a special nuclear material (SNM) and to define a standardized formatting scheme which will allow them to be incorporated into some of the modern, general-purpose Monte Carlo transport codes currently being used to simulate inspection techniques proposed for detecting fissionable material hidden in sea-going cargo containers. In this report, we will describe a Monte Carlo model for $\beta$-delayed $\gamma$-ray emission following the fission of SNM that can accommodate arbitrary time-dependent fission rates and photon collection histories. The model involves direct sampling of the independent fission yield distributions of the system, the branching ratios for decay of individual fission products and spectral distributions representing photon emission from each fission product and for each decay mode. While computationally intensive, it will be shown that this model can provide reasonably detailed estimates of the spectra that would be recorded by an arbitrary spectrometer and may prove quite useful in assessing the quality of evaluated data libraries and identifying gaps in the libraries. The accuracy of the model will be illustrated by comparing calculated and experimental spectra from the decay of short-lived fission products following the reactions ${ }^{235} \mathrm{U}\left(\mathrm{n}_{\mathrm{th}}, \mathrm{f}\right)$ and ${ }^{239} \mathrm{Pu}\left(\mathrm{n}_{\mathrm{th}}, \mathrm{f}\right)$. For general-purpose transport calculations, where a detailed consideration of the large number of individual $\gamma$-ray transitions in a spectrum may not be necessary, it will be shown that a simple parameterization of the $\gamma$-ray source function can be defined which provides high-quality average spectral distributions that should suffice for calculations describing photons being transported through thick attenuating media. Finally, a proposal for ENDF-compatible formats that describe each of the models and allow for their straightforward use in Monte Carlo codes will be presented.
\end{abstract}

\section{Introduction}

During the past few years there has been increasing interest in the development of new inspection techniques capable of detecting clandestine fissionable material that might be hidden in sea-going cargo containers ( $c f$. Slaughter, et al. [1] and references contained therein). Recent work by Norman, et al. [2] at Lawrence Berkeley National Laboratory has demonstrated that the relatively intense, high-energy $\left(E_{\gamma} \geq 2.5 \mathrm{MeV}\right), \beta$-delayed $\gamma$-rays emitted during the decay of short-lived fission products might prove to be a useful signature of fissionable material in the presence of thick hydrogenous and other cargoes. This, in turn, has identified the need to find a way to model delayed $\gamma$-ray spectra for a variety of fission rate histories and incorporate these models into modern, general-purpose Monte Carlo codes in order to assess their transport characteristics through a wide range of cargo compositions and geometries.

At the present time, no single evaluated data set exists that fully describes the production and temporal evolution of $\gamma$ rays from the decay of fission products; however, evaluated data sets containing all of the physical parameters required for such calculations do exist ( $c f$. England and Rider [3] and the NuDat nuclear database [4]). These data sets include estimates for the inde- 
pendent and cumulative fission yields of all fission products, branching ratios and lifetimes for the decay of ground and isomeric states and detailed tabulations of $\gamma$ rays emitted in their decay. In this report, we will describe a detailed Monte Carlo model that provides estimates of the delayed $\gamma$-ray spectra produced following neutron-induced fission of a special nuclear material (SNM) for any arbitrary fission rate and photon collection history. The model involves direct sampling of the independent fission yield distributions and decay properties of the individual fission products listed in England and Rider and NuDat. The accuracy of the model will be illustrated by comparing our calculated spectra with those reported by Norman, et al.

The spectrum of delayed $\gamma$-rays from the decay of an arbitrary short-lived $\left(t_{1 / 2} \leq 1-2 \mathrm{~min}\right)$ fission product tends to be composed of a large number of transitions, each with a relatively small absolute intensity per decay. This is especially true for the high-energy $\left(2.5 \mathrm{MeV} \leq E_{\gamma} \leq 8 \mathrm{MeV}\right)$ portion of the spectrum. As a result, the delayed $\gamma$-ray spectrum from the decay of a collection of short-lived fission products will be very complex indeed. Since attenuation coefficients for photons in most materials over this energy range are only weakly dependent on energy, there is often no real need to incorporate a highly-detailed delayed $\gamma$-ray source model into a Monte Carlo code in order to assess the effects of different attenuating media and geometries on the transmitted spectra. For this purpose, we will demonstrate that simple parametric fits to the source spectra provide high-quality, time-dependent, energy-averaged distributions that can be used in generalpurpose Monte Carlo photon transport calculations.

\section{Monte Carlo models of delayed $\gamma$-ray spectra}

A specific neutron-induced binary fission event and the subsequent decay of the associated fission fragments and their progeny can be represented as:

$$
n+{ }^{A} Z \rightarrow\left[\begin{array}{c}
{ }^{A_{1}} Z_{1} \rightarrow{ }^{A_{1}^{*}}\left(Z_{1}+1\right) \rightarrow{ }^{A_{1}}\left(Z_{1}+1\right) \rightarrow^{A_{1}}\left(Z_{1}+2\right) \cdots \\
A_{2} Z_{2} \rightarrow^{A_{2}-1}\left(Z_{2}+1\right)+n \rightarrow{ }^{A_{2}-1}\left(Z_{2}+2\right) \cdots
\end{array},\right.
$$

where ${ }^{A} Z$ is the target nucleus and ${ }^{A i} Z_{i}(i=1,2)$ represents the fission fragments following emission of prompt radiation but before $\beta$-decay or isomeric transitions have taken place. We indicate explicitly in Eqn. 1 that the nuclides we are dealing with may be nuclear isomers or $\beta$-delayed neutron emitters by the decay of the products ${ }^{\mathrm{Al}} \mathrm{Z}_{1}$ and ${ }^{\mathrm{A} 2} \mathrm{Z}_{2}$, respectively. The basic quantity that we wish to derive is the delayed gamma source function, $s_{\gamma}$, which describes the production and temporal evolution of photons following this event. Once $s_{\gamma}$ has been determined, the spectrum for any fission rate and decay (or counting) history can also be determined.

\subsection{Detailed (discrete) representation}

In its most general form, the delayed gamma source function can be defined as:

$$
s_{\gamma}\left(E, E_{\gamma}, t\right) \equiv \frac{d^{2} n_{\gamma}\left(E, E_{\gamma}, t\right)}{d t d E_{\gamma}},
$$


where $E$ is the energy of the fission-inducing particle (a neutron in our case), $E_{\gamma}$ is the energy of the delayed photon, $t$ is the time at which the photon is emitted following fission and $n_{\gamma}$ is the photon multiplicity (i.e. the number of photons emitted per event).

A detailed (discrete) representation of this source function can be generated by randomly choosing a fission product and its decay time following fission and then weighting its contribution by appropriate independent fission yield and lifetime values extracted from the England and Rider and NuDat data sets. For each decay, the energy of an emitted photon is chosen randomly from the known (observed) spectrum of photons emitted following decay of the fragment and the event is weighted according to the absolute intensity of the photon. There is no need to follow photon cascades directly; rather, we assume that the proper spectral distribution can be reproduced statistically by sampling individual photons from the known spectrum. Daughter production is determined by sampling from known branching ratios in the decay of the parent nuclide. Photon emission from decay of the daughter is determined in the same way as photon emission from the parent. The process is repeated for each of the progeny until a sufficiently long-lived nuclide is produced (e.g. we have chosen $t_{1 / 2}>10^{7} \mathrm{sec}$ as a cutoff).

The calculation of $s_{\gamma}$ can be fairly lengthy using this representation due to the large number of nuclides and decay $\gamma$ rays typically involved. For example, the independent yield evaluations of England and Rider and NuDat provide data for $\approx 1000$ nuclides, 100 isomers and 20,000 discrete $\gamma$-ray transitions. Nonetheless, the detailed Monte Carlo representation of a delayed $\gamma$-ray spectrum in histogram form with bin widths of $1 \mathrm{keV}$ that includes decay times in the range $0 \leq t$ $\leq 100 \mathrm{sec}$ following fission (and which possesses reasonable statistics for the more intense highenergy photons) takes only $\approx 10$ minutes to run on a $3-\mathrm{GHz}$ single-processor desktop PC. In the next section, calculations which approximately reproduce the irradiation and counting history of the experiments done by Norman, et al. will be discussed. These calculations have roughly three times better statistical quality (i.e. about ten times more counts per line) than the Norman, et al. experiments and yet took only $\approx 40$ minutes to run.

\subsection{Comparison with experiment}

Although more detailed benchmark experiments have yet to be carried out, we can get an initial estimate of how well the discrete model described above agrees with experiment by comparing to the data reported by Norman, et al. In these experiments, targets of ${ }^{235} \mathrm{U}$ (93\% isotopic abundance) and ${ }^{239} \mathrm{Pu}$ (95\% isotopic abundance) were exposed to a well-thermalized neutron flux for a period of $30 \mathrm{sec}$, after which they were counted with an $80 \%$ relative efficiency HPGe detector for $30 \mathrm{sec}$. The counting period was divided into 10 successive 3 -sec periods to follow the decay of individual lines. The transit time between the irradiation and counting locations was $\approx$ $2.5 \mathrm{sec}$. In order to acquire reasonable statistics, $\approx 20$ irradiation/counting cycles were performed for each target. The estimated time between each successive irradiation/counting cycle was $\approx 1-2$ min. For our purposes, we will assume that the time between successive irradiation/counting cycles was $100 \mathrm{sec}$ and that the transit time was $3 \mathrm{sec}$.

The fact that the relative and absolute efficiency functions for the detector used in the experiments of Norman, et al. are not well known posed a significant complication in terms of calculating estimates of the HPGe spectra. In addition, the axis of the detector was oriented perpendicular to the line connecting the source and detector rather than facing the source (i.e. "on axis") 
as might normally be expected. Also, the geometry of the shielding materials used by Norman, et al. was fairly complex. As a result, we have chosen to compare the predicted and experimental spectral distributions using the relative full-energy, single-escape and double-escape peak efficiency functions calculated by Owens, et al. [5] for a $7 \mathrm{~cm}$ (diameter) x $7 \mathrm{~cm}$ (length) $\mathrm{Ge}$ detector (this detector has a volume close to that of the detector actually used by Norman, et al.). Spectral magnitudes were normalized by reference to a strong, well-isolated line of known intensity. We note that the calculations of Owens, et al. are also for a source located on axis with the detector; therefore the energy dependence of the efficiency functions cannot be expected to reproduce the experiment exactly. A rough estimate of the uncertainties introduced by our use of the calculations in Owens, et al. can be obtained by referring to the efficiency functions reported by these authors for a $5 \mathrm{~cm}$ (diameter) x $5 \mathrm{~cm}$ (length) HPGe detector. For this smaller detector, the calculated full-energy peak efficiencies at $E_{\gamma}=2,3$, and $5 \mathrm{MeV}$, relative to that at $E_{\gamma}=1$ $\mathrm{MeV}$, are smaller than the corresponding ratios for the $7 \mathrm{~cm}$ (diameter) $7 \mathrm{~cm}$ (length) detector by $14 \%, 20 \%$ and $30 \%$, respectively. This implies uncertainties of $\approx 20-30 \%$ in our simple estimates of the full-energy peak efficiency at high energies.

The calculated and experimental spectra were normalized using the full-energy peak at 1427 $\mathrm{keV}$. This line is attributed to the decay of ${ }^{94} \mathrm{Sr}\left(t_{1 / 2}=75.3 \mathrm{sec}\right)$, which is sufficiently long-lived that timing uncertainties on the order of $1 \mathrm{sec}$ in the beginning of a counting period are not significant. Furthermore, the ${ }^{94} \mathrm{Rb}\left(t_{1 / 2}=2.70 \mathrm{sec}\right)$ precursor of this nuclide is sufficiently short-lived that uncertainties associated with precursor decay are not significant either. Finally, the independent and cumulative fission yields of ${ }^{94} \mathrm{Sr}$ have rather small uncertainties $(\leq 6 \%)$ in the thermal fission of ${ }^{239} \mathrm{Pu}$ and even smaller uncertainties for thermal fission of ${ }^{235} \mathrm{U}$. The actual normalization was done by comparing peak intensities at $1427 \mathrm{keV}$ in the 3 -sec spectrum from ${ }^{235} \mathrm{U}$ and ${ }^{239} \mathrm{Pu}$ beginning at $t_{0}=15 \mathrm{sec}$ following the end of an irradiation.

To proceed with our comparison between calculations and experiment, let $r\left(E_{\gamma}\right)$ represent the line spectrum incident upon the HPGe detector under experimental conditions, $o\left(E_{\gamma}\right)$ the complete spectrum registered by the detector and $m\left(E_{\gamma}\right)$ the line spectrum estimated from the Monte Carlo calculation. In the absence of any errors, the ratio $m\left(E_{\gamma}\right) / r\left(E_{\gamma}\right)$ should be a constant. The functions $o\left(E_{\gamma}\right)$ and $r\left(E_{\gamma}\right)$ are related by:

$$
o\left(E_{\gamma}\right)=\varepsilon_{f e p}\left(E_{\gamma}\right) r\left(E_{\gamma}\right)+\varepsilon_{1}\left(E_{\gamma}+m_{e} c^{2}\right) r\left(E_{\gamma}+m_{e} c^{2}\right)+\varepsilon_{2}\left(E_{\gamma}+2 m_{e} c^{2}\right) r\left(E_{\gamma}+2 m_{e} c^{2}\right)+b\left(E_{\gamma}\right),
$$

where $\varepsilon_{\text {fep }}, \varepsilon_{1}$ and $\varepsilon_{2}$ represent the full-energy, single-escape and double-escape peak efficiencies, respectively, and $b\left(E_{\gamma}\right)$ represents the continuum from the interaction of photons with energy $E_{\gamma}$. This implies that comparing the expected value $\left\langle o\left(E_{\gamma}\right)-b\left(E_{\gamma}\right)\right\rangle$ (estimated from experimental data) with the quantity

$$
\left\langle\varepsilon_{f e p}\left(e_{E_{\gamma}}\right) m\left(E_{\gamma}\right)+\varepsilon_{1}\left(E_{\gamma}+m_{e} c^{2}\right) m\left(E_{\gamma}+m_{e} c^{2}\right)+\varepsilon_{2}\left(E_{\gamma}+2 m_{e} c^{2}\right) m\left(E_{\gamma}+2 m_{e} c^{2}\right)\right\rangle
$$

(obtained from Monte Carlo calculations) should provide a reasonable first estimate of the quantitative agreement between calculation and experiment. The averaging implied by the brackets could be over any desired energy interval. In this case, we have taken the averages to be over individual lines or unresolved multiplets in the experimental spectrum. Adjacent energy bins with 
$(o(j)-b(j))>1.5^{*} \sigma_{\mathrm{b}}$ (see below) are assumed to comprise a single line or unresolved multiplet. The total number of counts in the a spectral peak is estimated as the sum of $(o(j)-b(j))$ over these bins and the centroid of the peak is estimated as the mean of $E_{\gamma}(j) *(o(j)-b(j))$. We note that the experimental measurements were taken with a system gain of $\approx 1.1 \mathrm{keV}$ per channel and also that the HPGe had a resolution of $\approx 2.0 \mathrm{keV}(\mathrm{FWHM})$ at $1332.5 \mathrm{keV}$.

The principal difficulty in making a reasonable comparison between calculated and experimental line spectra is the estimation of $b\left(E_{\gamma}\right)$. Rather than attempt to calculate this directly from first principles, we have chosen to use a simple approximation which should suffice for the present purposes. Because a very large number of lines contribute to the experimental spectra, $b\left(E_{\gamma}\right)$ tends to vary smoothly and rather slowly with energy. Motivated by this observation, we adopt a simple estimate for $b\left(E_{\gamma}\right)$ in the experimental spectra. First, the average number of counts in 50 adjacent energy bins (channels) is given by:

$$
\bar{o}=\frac{1}{50} \sum_{j_{0}}^{j_{0}+50} o(j),
$$

where $o(j)$ is the number of counts observed in the $j^{\text {th }}$ bin. In the absence of any a priori information on the fraction of total counts in the summation due to continuum events, we make the arbitrary but conservative assumption that the majority of counts are background; therefore the uncertainty in the background is approximately $\sigma_{\mathrm{b}}=\sqrt{\bar{o}}$. The average number of background counts per channel in this channel range, $\bar{b}$, can now be estimated as the average number of counts in each bin for which the relation $(o(j)-\bar{o})<2 * \sigma_{\mathrm{b}}$ is satisfied. This $\bar{b}$ is designated as $b\left(j_{0}+25\right)$ and the process is repeated for $j_{0}=0,50,100, \ldots$ until the whole experimental spectrum has been processed. Linear interpolation between the different $b\left(j_{0}+25\right)$ values can then be used to estimate $b\left(E_{\gamma}\right)$ for arbitrary energies.

Figures 1 and 2 show comparisons of the type described above for the case of thermal neutron incident on ${ }^{239} \mathrm{Pu}$ and photons collected in the $3 \mathrm{sec}$ bin beginning at $t_{0}=30 \mathrm{sec}$ following fission. Table 1 compares the calculated and experimental intensities of prominent $\gamma$-rays emitted with $1 \mathrm{MeV}<E_{\gamma}<5 \mathrm{MeV}$ collected in the $3 \mathrm{sec}$ bin beginning at $t_{0}=6 \mathrm{sec}$ following fission. Figures 3 and 4 show similar comparisons for the case of thermal neutron incident on ${ }^{235} \mathrm{U}$.

Scanning through the table and figures, it is immediately evident that the peak distributions and intensities are generally quite similar, although some obvious discrepancies are apparent. Some predicted lines in the spectra from both ${ }^{239} \mathrm{Pu}$ and ${ }^{235} \mathrm{U}$ fission products are not observed in the experimental spectra with significant intensity (and vice versa) and there are some notable discrepancies in the calculated and observed line intensities. There are a number of experimental uncertainties which can lead to appreciable errors in our calculations. For example, some fission yields from England and Rider have large uncertainties, prompt yields of isomeric states are typically uncertain to $\approx 50 \%$, normalization of $\gamma$-ray intensities is unknown for several nuclei with modest cumulative fission yields and there are certainly some high-lying lines that have not yet been seen in experiments.

The influence of uncertainties in isomeric yields, though likely not dominant, can in some cases be readily seen. For example, the intensities of the $1107 \mathrm{keV}$ and $1750 \mathrm{keV}$ lines are roughly twice as large in the calculated spectrum for ${ }^{239} \mathrm{Pu}$ at $t_{0}=6 \mathrm{sec}$ as in the experimental spectrum ( $c f$. Table 1). These lines arise with high intensity in the decay of ${ }^{96 \mathrm{~m}} \mathrm{Y}\left(t_{1 / 2}=9.6 \mathrm{sec}\right)$, 
but they are only weak components in the decay of ${ }^{96} \mathrm{Y}\left(t_{1 / 2}=5.34 \mathrm{sec}\right)$. Isomer ratios in fission have been notoriously difficult to measure or predict over the years and it may be that the ratios suggested by England and Rider need to be adjusted in this case. Such an adjustment would lie well within the uncertainties associated with their evaluated fission yields.

A careful study of fission yields derived from observed $\gamma$-ray transitions is described by Tipnis, et al. [6]. These authors used a helium-jet system to rapidly transfer fission products produced in ${ }^{235} \mathrm{U}\left(\mathrm{n}_{\mathrm{th}}, \mathrm{f}\right)$ reactions from a fission chamber to an HPGe detector. Overall, they found good agreement between their inferred independent yields and previously published studies; however, a number of discrepancies were noted. For example, it was found that the England and Rider estimate of the independent yield for ${ }^{96 \mathrm{~m}} \mathrm{Y}$ was too high by about a factor of two. A similarly detailed comparison between the calculated and experimental spectra of Norman, et al. is clearly unwarranted here because of the complications noted above; nonetheless, we can conclude that the general agreement is reasonable. Also, this method, coupled with accurately modeled benchmark spectra, may be very useful in assessing the general quality of the databases involved and may complement the approach described in [6]. It may also be possible to improve the quality of the evaluated data files where, at present, very large uncertainties exist.

\subsection{Parameterized (continuous) representation}

As noted earlier, there is often no need to incorporate a highly-detailed delayed $\gamma$-ray source model such as that described in Section 2.1 into a Monte Carlo code in order to assess the effects of different attenuating media and geometries on the transmitted spectra, particularly when the shielding materials involved have thicknesses of a few attenuation mean free paths or more. We have found that the delayed gamma source function can be well represented in these cases by a piecewise-continuous function of the form:

$$
s_{\gamma}\left(E, E_{\gamma}, t\right) \equiv A_{1}\left(E, E_{\gamma}\right) e^{-t / \tau_{1}\left(E, E_{\gamma}\right)}+A_{2}\left(E, E_{\gamma}\right) e^{-t / \tau_{2}\left(E, E_{\gamma}\right)}+A_{3}\left(E, E_{\gamma}\right) e^{-t / \tau_{3}\left(E, E_{\gamma}\right)},
$$

where the coefficients $A_{\mathrm{i}}$ and time constants $\tau_{\mathrm{i}}$ are defined over a given range of photon energies. Figure 5 shows representative examples of parameterized fits of this sort over $500-\mathrm{keV}$ intervals for the case of thermal neutrons incident on ${ }^{239} \mathrm{Pu}$ (the corresponding fitting parameters are given in Table 2). Note that these fits are only for times out to $\approx 100 \mathrm{sec}$ following fission and are probably not valid for much longer times. It is clear from the figure that the fits represent the $\gamma$ ray source functions quite accurately over this time range (similar quality fits were obtained for thermal neutrons incident on ${ }^{235} \mathrm{U}$ ). This simple model for $s_{\gamma}$ should permit relatively rapid transport calculations to be carried out over a wide range of conditions.

\section{Implementation in ENDF/B *}

The detailed representation of the delayed gamma source function described in Section 2.1 has already been incorporated into LLNL's Monte Carlo transport code "COG" [7] and is currently be tested in cargo interrogation scenarios; however, one of the primary goals of this LDRD

\footnotetext{
* Not recommended reading for the faint of heart or those with poor eyesight...
} 
project was to couch these models in a standardized format such as ENDF/B so that they can be used by any Monte Carlo transport code capable of reading that library (e.g. MCNP and its variants). To this end, we have recently proposed formatting schemes for both of our delayed gamma source function models to the Cross Section Evaluation Working Group (CSEWG) [8] for inclusion in the next version of ENDF/B. Our proposals were accepted and the formats should be included in the ENDF-6 manual [9] in the near future.

Turning now to the somewhat cryptic terminology of the ENDF-6 manual, if a material undergoes fission (LFI = 1), then an (unassigned) MT section may be used to specify the delayed gamma source function as either a continuous $(\mathrm{LO}=1)$ or discrete representation $(\mathrm{LO}=2)$ in an (unassigned) MF field. The detailed structure of each of these source specifications is outlined in Sections 3.1 and 3.2 below.

\subsection{Option $1(\mathrm{LO}=1)$ : Continuous representation}

In the continuous representation of the delayed gamma source function, $s_{\gamma}\left(E, E_{\gamma}, t\right)$ may be defined as a weighted sum of exponentials ( $c f$. Eqn. 6):

$$
s_{\gamma}\left(E, E_{\gamma}, t\right) \equiv \sum_{i=1}^{N C} A_{i}\left(E, E_{\gamma}\right) \operatorname{Exp}\left(-t / \tau_{i}\left(E, E_{\gamma}\right)\right)
$$

where NC is the number of terms, $A_{\mathrm{i}}\left(E, E_{\gamma}\right)$ is the weight of the $i^{\text {th }}$ term and $\tau_{\mathrm{i}}\left(E, E_{\gamma}\right)$ is the corresponding time constant. Note that the weights in Eqn. 7 must be normalized so that the photon spectrum satisfies the constraint:

$$
\frac{d n_{\gamma}\left(E, E_{\gamma}\right)}{d E_{\gamma}}=\sum_{i=1}^{N C} A_{i}\left(E, E_{\gamma}\right) \tau_{i}\left(E, E_{\gamma}\right) .
$$

The total photon multiplicity in this case is given by:

$$
n_{\gamma}(E)=\sum_{i=1}^{N C} \int_{0}^{E_{\gamma}^{\max }} A_{i}\left(E, E_{\gamma}\right) \tau_{i}\left(E, E_{\gamma}\right) d E_{\gamma}
$$

The coefficients, $A_{\mathrm{i}}\left(E, E_{\gamma}\right)$, and time constants, $\tau_{\mathrm{i}}\left(E, E_{\gamma}\right)$, are tabulated on a two-dimensional grid using a format very similar to the Arbitrary Tabulated Function option (LF $=1)$ used for the energy distributions of emitted particles $(\mathrm{MF}=5)$. In our proposed format, both the exponential coefficients and time constants are tabulated as functions of the incident projectile energy, $E$, and outgoing photon energy, $E_{\gamma}$. If we define the following parameters:

$$
\begin{aligned}
& \mathrm{NC}=\text { number of exponential decay terms (note that } \mathrm{NC}=3 \text { in Eqn. } 6 \text { ), } \\
& \mathrm{NR}=\text { number of interpolation ranges for the incident projectile energy, } \\
& \mathrm{NE}=\text { number of incident energy points at which the distribution is given, } \\
& \mathrm{NF}=\text { number of secondary energy points in the tabulation, } \\
& \mathrm{Eint}=\text { energy interpolation scheme (see ENDF-6 manual, section } 0.6 .2 \text { ), }
\end{aligned}
$$




$$
\begin{aligned}
& A_{\mathrm{i}}\left(E, E_{\gamma}\right)=\text { coefficient of the } i^{\text {th }} \text { exponential term }[1 /(\mathrm{sec}-\mathrm{eV})], \\
& \tau_{\mathrm{i}}\left(E, E_{\gamma}\right)=\text { time constant for the } i^{\text {th }} \text { exponential term }[\mathrm{sec}]
\end{aligned}
$$

then the structure of this section may be written as:

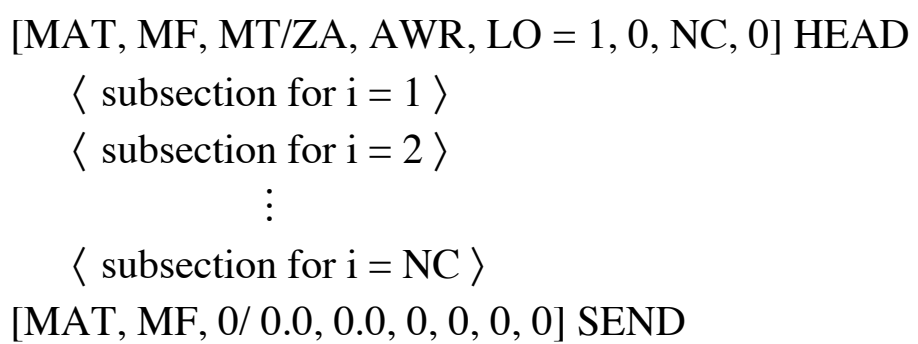

where the structure of each subsection is:

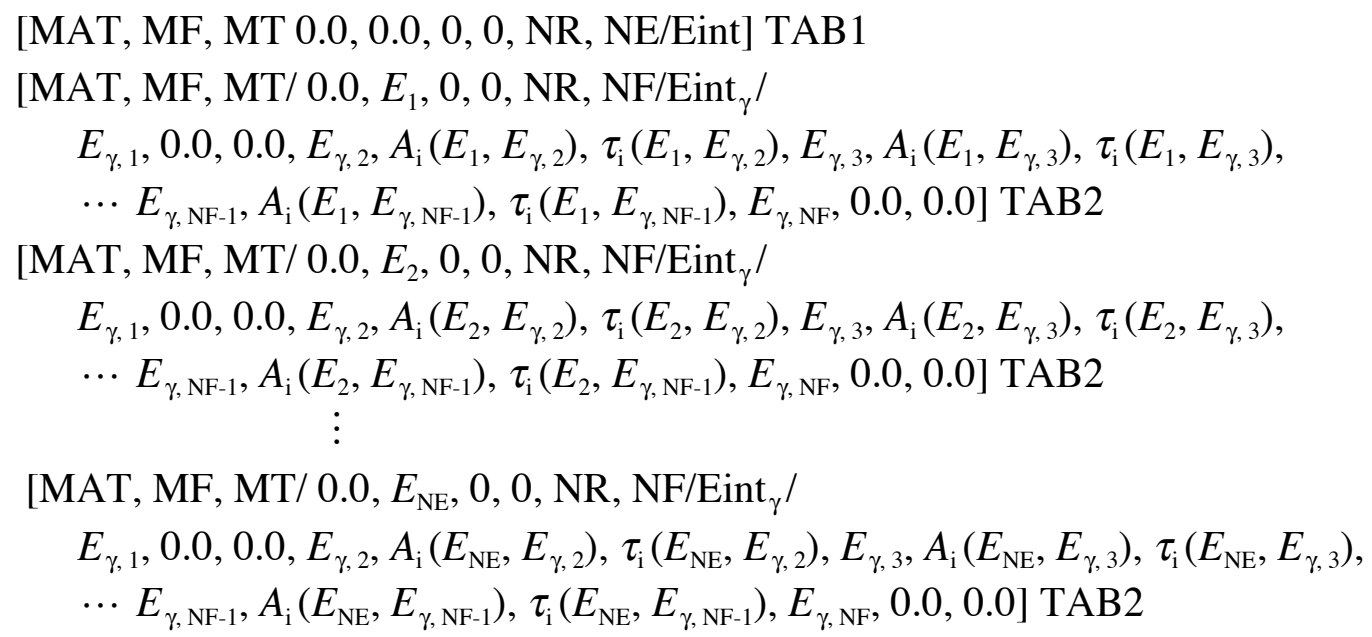

\subsection{Option $2(\mathrm{LO}=2)$ : Discrete representation}

In the discrete representation of the delayed gamma source function, $s_{\gamma}\left(E, E_{\gamma}, t\right)$ may be defined as a sum over a series of delta functions in photon energy:

$$
s_{\gamma}\left(E, E_{\gamma}, t\right) \equiv \sum_{i=1}^{N G(E)} \delta\left(E_{\gamma}-E_{\gamma, i}\right) s_{\gamma, i}(E, t)
$$

where $N G(E)$ is number of photon lines observed following fission induced by a projectile at incident energy $E$. The discrete source function for the $i^{\text {th }}$ photon, $s_{\gamma, \mathrm{i}}(E, t)$, is defined as:

$$
s_{\gamma, i}(E, t) \equiv \frac{d n_{\gamma, i}(E, t)}{d t}
$$

and the total photon multiplicity is given by: 


$$
n_{\gamma}(E)=\sum_{i=1}^{N G(E)} \int_{0}^{\infty} s_{\gamma, i}(E, t) d t
$$

Since the discrete photon source function depends on both the incident projectile energy and the time since the fission event, it is not like any existing ENDF/B data type. In our proposed format, each photon line $i$ is associated with a two-dimensional table representing $s_{\gamma, \mathrm{i}}(E, t)$. The table is designed to interpolate over the incident projectile energy, $E$, and the time since the fission event, $t$. If we define the following parameters:

$$
\begin{aligned}
& \mathrm{NG}=\text { number of observed photon lines (may be large), } \\
& \mathrm{NR}=\text { number of interpolation ranges for the incident projectile energy, } \\
& \mathrm{NE}=\text { number of incident energy points at which the distribution is given, } \\
& \text { Eint }=\text { energy interpolation scheme (see ENDF-6 manual, section 0.6.2), } \\
& \mathrm{NT}=\text { number of time points in the tabulation (may be large), } \\
& \text { Tint }=\text { time interpolation scheme (see ENDF-6 manual, section } 0.6 .2 \text { ), } \\
& s_{\gamma, \mathrm{i}}(E, t) \quad=\text { discrete source function for the } i^{\text {th }} \text { photon, }
\end{aligned}
$$

then the structure of this section may be written as:

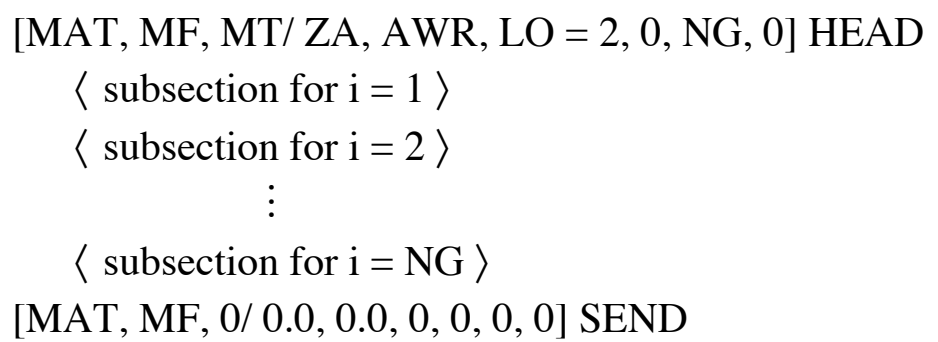

where the structure of each subsection is:

[MAT, MF, MT/ 0.0, 0.0, 0, 0, NR, NE/Eint] TAB1

[MAT, MF, MT/ 0.0, $E_{1}, 0,0, \mathrm{NR}, \mathrm{NT} / \mathrm{Tint} /$

$$
\begin{aligned}
& t_{1}, 0.0, t_{2}, \mathrm{~s}_{\gamma, \mathrm{i}}\left(E_{1}, t_{2}\right), t_{3}, \mathrm{~s}_{\gamma, \mathrm{i}}\left(E_{1}, t_{3}\right), \\
& \left.\cdots t_{\mathrm{NT}-1}, \mathrm{~s}_{\gamma, \mathrm{i}}\left(E_{1}, t_{\mathrm{NT}-1}\right), t_{\mathrm{NT}}, 0.0\right] \mathrm{TAB} 2
\end{aligned}
$$

[MAT, MF, MT/ 0.0, $E_{2}, 0,0, \mathrm{NR}, \mathrm{NT} / \mathrm{Tint} /$

$$
\begin{aligned}
& t_{1}, 0.0, t_{2}, \mathrm{~s}_{\gamma, \mathrm{i}}\left(E_{2}, t_{2}\right), t_{3}, \mathrm{~s}_{\gamma, \mathrm{i}}\left(E_{2}, t_{3}\right), \\
& \left.\cdots t_{\mathrm{NT}-1}, \mathrm{~s}_{\gamma, \mathrm{i}}\left(E_{2}, t_{\mathrm{NT}-1}\right), t_{\mathrm{NT}}, 0.0\right] \mathrm{TAB} 2
\end{aligned}
$$

[MAT, MF, MT/ 0.0, $E_{\mathrm{NE}}, 0,0, \mathrm{NR}, \mathrm{NT} / \mathrm{Tint} /$

$$
\begin{aligned}
& t_{1}, 0.0, t_{2}, \mathrm{~s}_{\gamma, \mathrm{i}}\left(E_{\mathrm{NE}}, t_{2}\right), t_{3}, \mathrm{~s}_{\gamma, \mathrm{i}}\left(E_{\mathrm{NE}}, t_{3}\right), \\
& \left.\cdots t_{\mathrm{NT}-1}, \mathrm{~s}_{\gamma, \mathrm{i}}\left(E_{\mathrm{NE}}, t_{\mathrm{NT}-1}\right), t_{\mathrm{NT}}, 0.0\right] \mathrm{TAB} 2
\end{aligned}
$$




\section{Summary and comments}

Prior to the execution of this LDRD, complete end-to-end (source-to-detector) Monte Carlo simulations of neutron interrogation schemes that involved inducing fission in SNM and detecting $\beta$-delayed $\gamma$ rays from the resulting fission products (e.g. Slaughter, et al.) were not directly possible because appropriate data libraries describing the production and temporal evolution of these photons were not coupled into the codes. In this report, we have proposed two different models for the delayed gamma source function that can be readily incorporated into modern, general-purpose Monte Carlo transport codes and used for such applications. The first is a detailed representation which involves tabulating the source function on a discrete time/energy grid. Since the grid can be made almost arbitrarily fine, this representation can be used to generate detailed spectral information if needed. The second is a parameterized representation which is much more compact and based on simple continuous functions. It is similar in spirit to currentlyused descriptions of $\beta$-delayed neutron emission following fission and should be useful when detailed spectral information is not really needed. Thus far, parameters describing these two representations have been calculated for thermal, fission-spectrum and $14 \mathrm{MeV}$ neutron-induced fission of both ${ }^{235} \mathrm{U}$ and ${ }^{239} \mathrm{Pu}$. The detailed representation of the source function has already been incorporated into LLNL's Monte Carlo transport code "COG" and schemes have been proposed and accepted for describing both models in ENDF-compatible formats so that they can be used by any Monte Carlo transport code capable of reading that library.

The reader is cautioned that the results obtained from Monte Carlo calculations are limited by the physics contained in the code being used and the quality of the experimental data contained in the libraries from which the code draws its information. For the most part, the decay properties of the longer-lived fission products are well defined for most applications; however, for the case of interrogating cargo for the presence of clandestine fissionable material, we must deal with some of the shortest-lived fission products with $\mathrm{Z} \leq \mathrm{Z}_{\mathrm{p}}$ (the most probable atomic number of a fission fragment isobar), where data sets on decay radiations may be incomplete and where estimated uncertainties in independent fission yields are often rather large. The adequacy of the evaluated data libraries can only be judged through a comparison of detailed benchmark experiments with Monte Carlo calculations. Such benchmark experiments have recently been done at Lawrence Berkeley National Laboratory [10] and may add to information gained from other experimental methods (e.g. [6]) for determining fission-fragment yields.

Finally, we note that this report is essentially a synopsis of two papers published during the term of this LDRD (Pruet, et al. [11] and Brown, et al. [12]). Posters describing this work and its application to the simulation of cargo inspection techniques have also been presented at technical conferences (Pruet, et al. [13] and Descalle, et al. [14]) and a comprehensive discussion of the utility of delayed gamma source models such as those presented here in end-to-end (source-todetector) neutron and photon transport calculations in sea-going cargo containers is currently in press with the Journal of Applied Physics (Pruet, et al. [15]).

\section{Acknowledgements}

We would like to thank Richard Buck and Edward Lent of LLNL for many helpful discussions on how to incorporate the delayed gamma source models presented here into modern 
Monte Carlo transport codes and Dermott Cullen of LLNL for his expert advice on how to develop formatting schemes to describe the models and successfully present them to the CSEWG for inclusion in ENDF/B. Finally, we would like to thank Rosemary Walling of LLNL for initiating this LDRD project and securing the original funding for it from the Nonproliferation, Arms Control and International Security (NAI) Directorate.

This work was performed under the auspices of the U.S. Department of Energy by the University of California, Lawrence Livermore National Laboratory under contract W-7405-ENG-48.

\section{References}

[1] D. Slaughter, M. Accatino, A. Bernstein, J. Candy, A. Dougan, J. Hall, A. Loshak, D. Manatt, A. Meyer, B. Pohl, S. Prussin, R. Walling and D. Weirup, "Detection of special nuclear material in cargo containers using neutron interrogation", LLNL Internal Report UCRL-ID-155315 (2003).

[2] E. Norman, S. Prussin, R.-M. Larimer, H. Shugart, E. Browne, A. Smith, R. McDonald, H. Nitsche, P. Gupta, M. Frank and B. Gosnell, "Signatures of special nuclear material: highenergy $\gamma$-rays following fission", Nuclear Instruments and Methods B (in press).

[3] T. England and B. Rider, "Evaluation and compilation of fission product yields", LANL Internal Report LA-UR-94-3106, ENDF-349 (1994).

[4] NuDat is a web-based nuclear data archive maintained by the National Nuclear Data Center (NNDC) at BNL. The URL is http://www.nndc.bnl.gov/nddc/nudat/.

[5] A. Owens, N. Gehrels, S. Pascarelle and B. Teegarden "Monte Carlo calculations of Ge detector escape-peak efficiencies”, IEEE Transactions on Nuclear Science 38 (1991) 559.

[6] S. Tipnis, J. Campbell, G. Couchell, S. Li, H. Nguyen, D. Pullen, W. Schier, E. Seabury and T. England, "Yields of short-lived fission products produced following ${ }^{235} \mathrm{U}\left(\mathrm{n}_{\mathrm{th}}, \mathrm{f}\right)$ ", Phys. Rev. C 58 (1998) 905.

[7] R. Buck and E. Lent, LLNL, private communication.

[8] CSEWG is a collaborative effort between national laboratories, industry and universities in the United States and Canada responsible for producing and maintaining the U.S. Evaluated Nuclear Data File ENDF/B. The URL is http://www.nndc.bnl.gov/csewg/index.html.

[9] The internationally agreed-upon format for evaluated nuclear reaction data files is specified by the ENDF-6 manual, available as document IAEA-NDS-76, Rev. 6 (this is a reprint of report ENDF-102, edited by P. Rose and C. Dunford, July 1990). The URL is http://wwwnds.iaea.org/indg_endfor.html.

[10] E. Norman (LLNL) and S. Prussin (UCB), private communication.

[11] J. Pruet, J. Hall, M.-A. Descalle and S. Prussin, "Monte Carlo models for the production of $\beta$-delayed gamma-rays following fission of special nuclear materials", LLNL Internal Report UCRL-JRNL-202168 (2004) and Nuclear Instruments and Methods B 222 (2004) 403.

[12] D. Brown, J. Pruet, G. Hedstrom, J. Hall and M.-A. Descalle, "Proposal for ENDF formats that describe emission of post-fission beta-delayed photons", LLNL Internal Report UCRL-TR-206607 (2004).

[13] J. Pruet, J. Hall, M.-A. Descalle and S. Prussin, "Delayed gamma rays produced following fission: modeling and comparison with experiments", LLNL Internal Report UCRL-POST- 
207029 (2004), poster presentation at the $18^{\text {th }}$ International Conference on the Application of Accelerators in Research and Industry (CAARI), Fort Worth, TX, October 10-15, 2004.

[14] M.-A. Descalle, J. Pruet, J. Hall, S. Prussin and D. Slaughter, "Neutron interrogation of containers: Monte Carlo simulations for various cargo and beam configurations", LLNL Internal Report UCRL-POST-207121 (2004), poster presentation at the $18^{\text {th }}$ International Conference on the Application of Accelerators in Research and Industry (CAARI), Fort Worth, TX, October 10-15, 2004.

[15] J. Pruet, M.-A. Descalle, J. Hall, B. Pohl and S. Prussin "Neutron and photon transport in sea-going cargo containers", LLNL Internal Report UCRL-JRNL-206586 (2004) and Journal of Applied Physics (in press). 

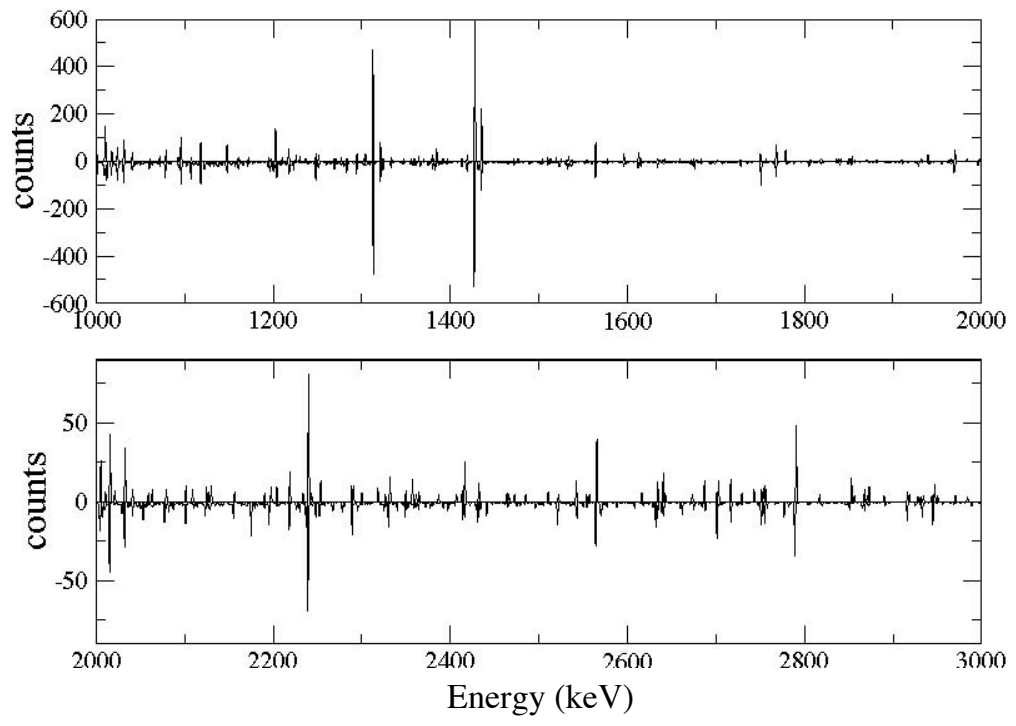

Figure 1: Comparison between the experiments of Norman et al. and the present calculations for thermal neutrons incident on ${ }^{239} \mathrm{Pu}$ and photons in the 1-3 MeV range collected in the $3 \mathrm{sec}$ bin beginning at $t_{0}=30 \mathrm{sec}$ following fission. The experimental line spectrum, including full-energy, single-escape and double-escape peaks, is shown in the upper half of each panel and the corresponding Monte Carlo result multiplied by -1 is shown in the bottom half.
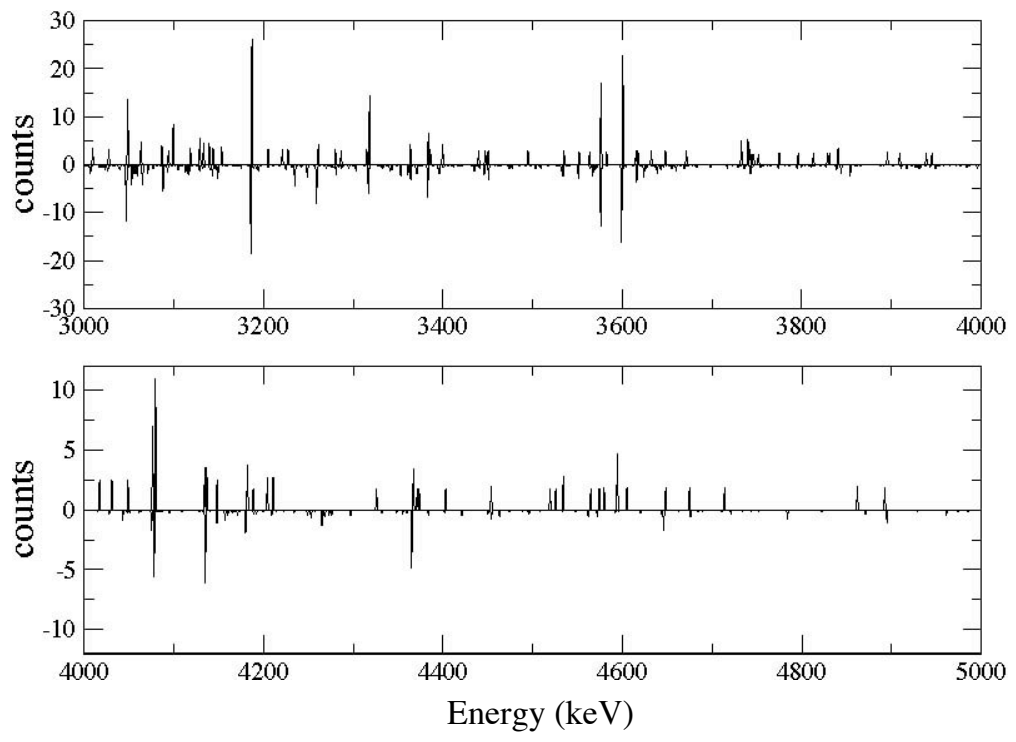

Figure 2: Same as Fig. 1 except for photons in the 3-5 MeV range. 
Table 1: Prominent lines observed in the decay spectra for thermal neutrons incident on ${ }^{239} \mathrm{Pu}$ at $t_{0}=6 \mathrm{sec}$

\begin{tabular}{|c|c|c|c|}
\hline \multirow{2}{*}{$\frac{E_{\gamma}(\mathrm{keV})}{1427.0}$} & Experimental counts ${ }^{a}$ & \multicolumn{2}{|c|}{ Monte Carlo counts } \\
\hline & $657.4[13.4]$ & 680.6 & {$[5.9]$} \\
\hline 1312.6 & $587.7 \quad[12.0]$ & 614.3 & {$[5.4]$} \\
\hline 1750.3 & $256.1 \quad[5.2]$ & 535.4 & [4.7] \\
\hline 1107.0 & $136.6[2.8]$ & 382.8 & {$[3.3]$} \\
\hline 1010.0 & $233.5[4.8]$ & 225.3 & {$[2.0]$} \\
\hline 1118.0 & $185.7 \quad[3.8]$ & 183.6 & [1.6] \\
\hline 1022.0 & $161.9 \quad[3.3]$ & 200.7 & {$[1.8]$} \\
\hline 1218.0 & $207.0 \quad[4.2]$ & 128.8 & [1.1] \\
\hline 1435.0 & 201.6 & 118.3 & {$[1.0]$} \\
\hline 1278.7 & $58.2 \quad[1.2]$ & 196.2 & {$[1.7]$} \\
\hline 2237.0 & $105.2[10.0]$ & 114.0 & {$[5.5]$} \\
\hline 2789.0 & $57.5 \quad[5.5]$ & 44.4 & {$[2.1]$} \\
\hline 2564.0 & $31.0[2.9]$ & 34.5 & [1.7] \\
\hline 2701.0 & $30.1[2.9]$ & 32.6 & [1.6] \\
\hline 2012.5 & $14.1 \quad[1.3]$ & 48.5 & {$[2.3]$} \\
\hline 2175.0 & $36.4[3.5]$ & 24.0 & [1.1] \\
\hline 2716.7 & $24.1 \quad[2.3]$ & 25.4 & [1.2] \\
\hline 2330.0 & $28.0 \quad[2.7]$ & 19.6 & [0.9] \\
\hline 2945.0 & $27.0 \quad[2.6]$ & 19.4 & [0.9] \\
\hline 2077.0 & [1.6] & 27.7 & {$[1.3]$} \\
\hline 2247.2 & {$[2.0]$} & 24.0 & [1.1] \\
\hline 3287.0 & $20.0 \quad[5.9]$ & 54.5 & {$[10.3]$} \\
\hline 3188.2 & $45.0[13.4]$ & 0.0 & {$[0.0]$} \\
\hline 3399.7 & $3.8 \quad[1.1]$ & 39.4 & [7.5] \\
\hline 3599.0 & $11.8 \quad[3.5]$ & 20.1 & {$[3.8]$} \\
\hline 3259.0 & [4.9] & 12.9 & [2.4] \\
\hline 3184.0 & {$[1.2]$} & 24.5 & [4.6] \\
\hline 3576.0 & {$[4.3]$} & 13.5 & [2.6] \\
\hline 3402.8 & [6.6] & 0.0 & {$[0.0]$} \\
\hline 3047.0 & {$[1.5]$} & 13.9 & [2.6] \\
\hline 3616.0 & $7.8 \quad[2.3]$ & 7.8 & [1.5] \\
\hline 4078.0 & $14.3[11.4]$ & 6.6 & {$[6.3]$} \\
\hline 4073.1 & $6.5 \quad[5.2]$ & 4.7 & {$[4.5]$} \\
\hline 4265.0 & $7.5 \quad[6.0]$ & 2.2 & [2.1] \\
\hline 4135.0 & $3.0 \quad[2.4]$ & 5.5 & {$[5.2]$} \\
\hline 4363.9 & $2.4 \quad[1.9]$ & 6.0 & [5.7] \\
\hline 4227.5 & $4.3 \quad[3.4]$ & 0.0 & {$[0.0]$} \\
\hline
\end{tabular}

\footnotetext{
${ }^{a}$ Numbers in square brackets are the percentage contributions of each line to the total number of photo-peak counts in $1 \mathrm{MeV}$ bins. For example, we estimate there to be $\approx 4970$ (experimental) photo-peak counts in the range $1-2 \mathrm{MeV}$, so the line at $1427 \mathrm{keV}$ contributes $\approx 13.4 \%$ to the total. The discrepancy between the normalizations of the experimental and Monte Carlo results is due to the fact that $\approx 60 \%$ of the photon counts come from very weak lines. These lines are counted as part of the "background" in the analysis of the experimental data.
} 

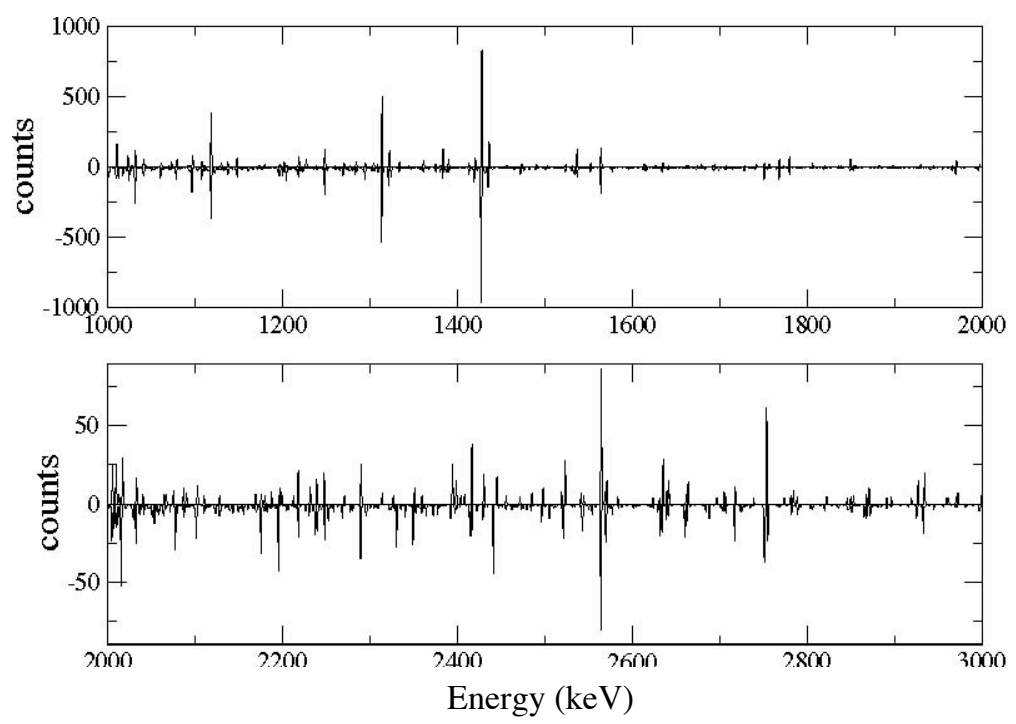

Figure 3: Comparison between the experiments of Norman et al. and the present calculations for thermal neutrons incident on ${ }^{235} \mathrm{U}$ and photons in the $1-3 \mathrm{MeV}$ range collected in the $3 \mathrm{sec}$ bin beginning at $t_{0}=30 \mathrm{sec}$ following fission. The experimental line spectrum, including full-energy, single-escape and double-escape peaks, is shown in the upper half of each panel and the corresponding Monte Carlo result multiplied by -1 is shown in the bottom half.
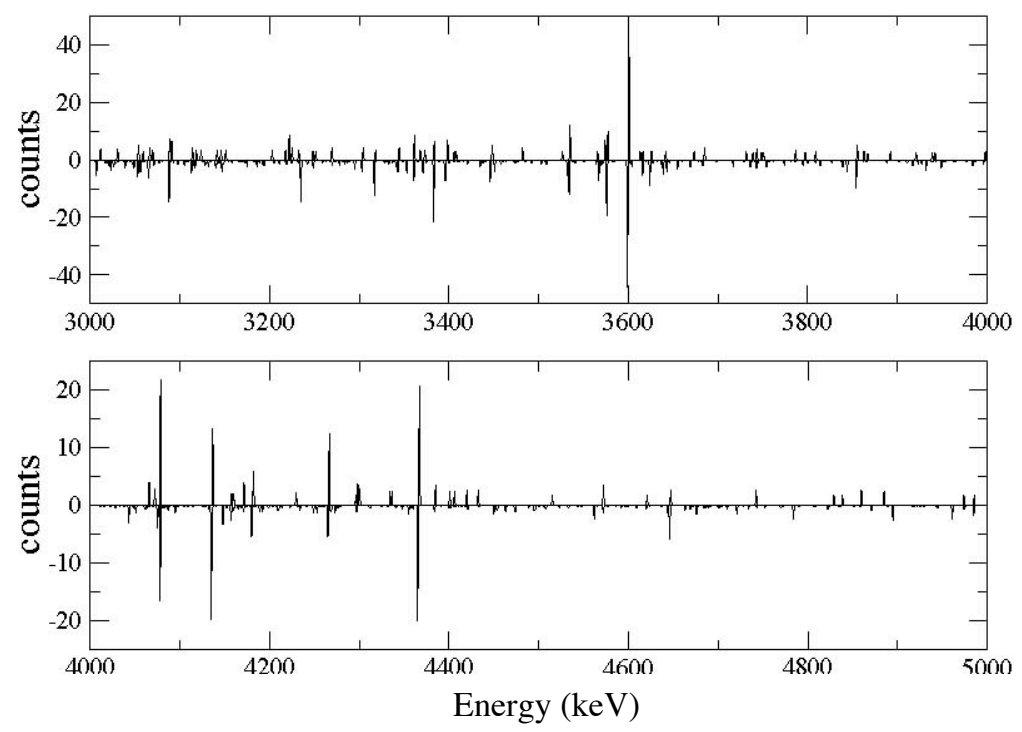

Figure 4: Same as Fig. 3 except for photons in the 3-5 MeV range. 


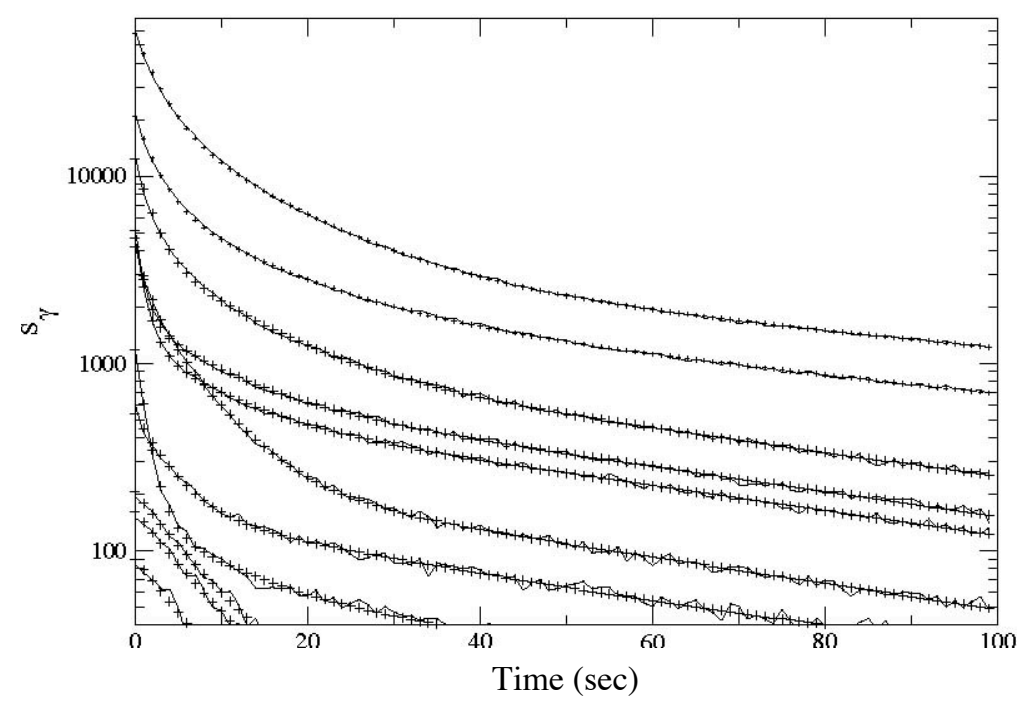

Figure 5: Comparison between the Monte Carlo calculation of the delayed gamma source function and simple fits to the function based on Eqn. 6 for thermal neutrons incident on ${ }^{239} \mathrm{Pu}$. The different lines correspond to different energy intervals. The top line (highest photon counts) represents the photon source function in the range 500-1000 $\mathrm{keV}$, the next line in the range 1000-1500 keV, and so on until the lowest line which represents counts in the 5500$6000 \mathrm{keV}$ range. The thin lines correspond to the Monte Carlo calculations and the crosses correspond to the simple fits described by Eqn. 6 . The units for the source function are photons/( $\sec \times 10^{6}$ fissions $\left.\times 500 \mathrm{keV}\right)$.

Table 2: Fit parameters for $s_{\gamma}$ for thermal neutrons incident on ${ }^{239} \mathrm{Pu}$

\begin{tabular}{rcccccc}
\hline \multicolumn{1}{c}{$E_{\gamma}(\mathrm{keV})$} & $A_{1}$ & $\tau_{1}(\mathrm{sec})$ & \multicolumn{1}{c}{$A_{2}$} & $\tau_{2}(\mathrm{sec})$ & $A_{3}$ & $\tau_{3}(\mathrm{sec})$ \\
\hline $500-1000$ & $3.63 \mathrm{E}+04$ & 2.61 & $1.85 \mathrm{E}+04$ & 12.09 & $3.34 \mathrm{E}+03$ & 97.73 \\
$1000-1500$ & $1.32 \mathrm{E}+04$ & 2.34 & $5.63 \mathrm{E}+03$ & 12.31 & $2.13 \mathrm{E}+03$ & 87.82 \\
$1500-2000$ & $8.12 \mathrm{E}+03$ & 1.81 & $3.17 \mathrm{E}+03$ & 10.35 & $1.05 \mathrm{E}+03$ & 69.21 \\
$2000-2500$ & $3.40 \mathrm{E}+03$ & 0.95 & $1.04 \mathrm{E}+03$ & 8.61 & $7.00 \mathrm{E}+02$ & 65.43 \\
$2500-3000$ & $3.31 \mathrm{E}+03$ & 1.08 & $8.09 \mathrm{E}+02$ & 7.84 & $5.52 \mathrm{E}+02$ & 65.61 \\
$3000-3500$ & $2.10 \mathrm{E}+03$ & 5.92 & $1.86 \mathrm{E}+03$ & 1.39 & $2.42 \mathrm{E}+02$ & 61.93 \\
$3500-4000$ & $3.76 \mathrm{E}+02$ & 3.79 & $1.46 \mathrm{E}+02$ & 43.69 & $1.67 \mathrm{E}+01$ & $5.47 \mathrm{E}+06$ \\
$4000-4500$ & $1.02 \mathrm{E}+03$ & 1.25 & $9.89 \mathrm{E}+01$ & 10.30 & $5.55 \mathrm{E}+01$ & 86.24 \\
$4500-5000$ & $1.78 \mathrm{E}+02$ & 6.64 & $2.37 \mathrm{E}+01$ & 34.24 & $2.29 \mathrm{E}+00$ & $2.00 \mathrm{E}+06$ \\
$5000-5500$ & $1.41 \mathrm{E}+02$ & 6.76 & $1.72 \mathrm{E}+01$ & 28.83 & $2.78 \mathrm{E}+00$ & $3.50 \mathrm{E}+07$ \\
$5500-6000$ & $2.96 \mathrm{E}+02$ & 10.21 & $1.29 \mathrm{E}+02$ & 18.71 & $-3.36 \mathrm{E}+02$ & 13.83 \\
\hline
\end{tabular}

